\title{
Housing Investment Model under High Housing Prices in Beijing Xinfang Song ${ }^{a}$ and Shaoqiang Wang $^{b}$
}

General Ability Teaching Department, Beijing Information Technology College, Beijing, 100015 asongxf@bitc.edu.cn, bwangsq@bitc.edu.cn

\begin{abstract}
In this paper, we establish a mathematical model of ordinary residents in Beijing to invest in the choice of a house or choose to rent a suitable problem. First of all, from the actual economic situation in Beijing, we dig out the five impact factors of residents in Beijing that affect people to buy or rent a house, namely: family income, rental income, savings and loan interest rates, housing depreciation rate, housing vacancy rate. Secondly, from the perspective of ordinary residents of loans to buy a house, buy a house and buy a house rental of the three options of the optimal problem. secondly, we establishes a linear programming model to analyze the optimal solution of a house or a rental. By comparing the appreciation of the property and the rental ratio, the relationship between house price and rent is studied, and the specific and suitable choice for the purchase of the house is suitable.
\end{abstract}

Keywords: Family income; Rental ratio; Real estate appreciation; Engel coefficient; Happiness index

\section{北京高房价形势下住房投资模型的探索研究}

\author{
宋新芳 王少强 \\ (北京信息职业技术学院通用能力教学部, 北京, 100015)
}

摘要: 本文通过建立数学模型研究了普通居民在北京投资时选择买房还是选择租房合适的问题。首先本文从北京的实际 经济情况出发, 找出影响北京人选择买房或租房的 5 点重要因素, 分别为: 家庭收入、租金收入、储蓄及贷款利率、房屋折 旧率、房屋空置率。其次从普通居民角度分析贷款买房、储蓄买房以及买房出租的三种选择的最优问题。最后建立线性规划 模型分析买房或者租房的最优解, 通过对房产升值与租售比进行作图比较, 研究房价与租金的关系, 并根据结果分析做出了 具体而又详实的买房合适还是租房合适的最优选择。

关键词: 家庭收入; 租售比; 房产升值; 恩格尔系数; 幸福指数

分类号: AMS(2000) 76Z05 中图分类号: O212 文献标识码: A

引言

近几年北京市房价持续上涨，高昂的房价给北京带来了一系列的社会与经济问题。作为普通居民在北 京选择买房还是租房是困扰很多人的问题。如果从租售比的角度看, 北京房价过高, 存在泡沫, 因此理性 的角度应该是租房, 但是, 从投资角度看, 房产的增值情况较好, 在北京适合投资, 而租房却是消费。本 文通过查找相关文献找出影响北京人买房或者租房的主要相关影响因素, 通过建立规划模型找出最优解得 到了北京选择买房或租房的选择。

北京市作为我们国家的政治、经济、文化中心, 其生活质量在全国一直处于领先地位。所以, 人们为 了更为舒适的生活大多数都会进行住房投资。但是，住房的投资受到各方面因素的影响。比如：储蓄及贷 款利率、家庭收入、房价、房屋折旧率等。所以, 本文讨论贷款买房和储蓄买房两种买房方式下成本和收 益的相对关系, 以及买房出租的住房投资方式。为此, 我们建立了这三种决策下用户成本和收益的函数关 
系。通过此函数关系来求解住房投资的问题。

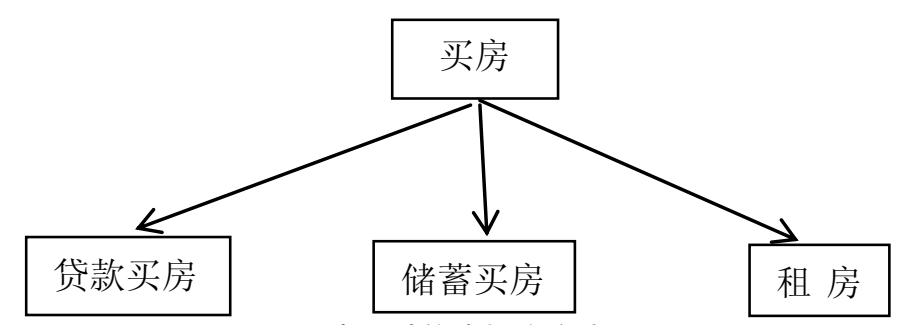

图 1 北京三种住房投资方式

\section{1 贷款买房和储蓄买房的模型}

假设北京市普通居民打算贷款买房，为研究贷款买房的最优解建立如下模型：假设家庭每年的总收入 为 $\mathrm{A}$, 家庭储蓄金额为 $\mathrm{B}$, 且储蓄金额 $\mathrm{B}$ 为固定值, 住房价格为 $Q_{a}$, 住房消费量为 $Z_{a}$, 除住房外的其他 消费量为 $Z_{b}$, 一方面这部分固定储蓄金额可以用于购买房产, 另一方面也可以用于金融投资(如存到银行 获取利息），假设固定储蓄金额每年可以产生的收入利率为 c。

如果一个家庭租房，所面临的预算约束条件是：

$Q_{b} Z_{b}+d Q_{a} Z_{a}=A+B \times C$

如果一个家庭想购房, 则所面临的预算约束条件是:

$Q_{b} Z_{b}+d Q_{a} Z_{a}+N n=A+(B-D) \times C$

则 $Q_{a} Z_{a}=N+D$

$D \leq B$

(公式中: $D$ 为首付款, $N$ 为本金, $n$ 为抵押贷款的利率, $d$ 为每年用于住房的各项费用占房价的比率。) 使用储蓄减少抵押贷款和使用储蓄获取利息是不同的两种情况, 即: 用储蓄购房时, 成本 $d_{1}=Q_{a}\left[c\left(1-v_{1}\right)+d\right]$ 。

用储蓄抵押贷款购房时, 成本 $d_{2}=Q_{a}(n+d)$ 。其中年利率 $\mathrm{c}$ 和各年抵押贷款利率 $\mathrm{n}$ 如图 2 所示:

\begin{tabular}{|c|c|c|}
\hline \multicolumn{3}{|c|}{ 利率调整表 } \\
\hline & 调整后利率(单位: \%) & 调整前利率(单位: \%) \\
\hline \multicolumn{3}{|c|}{ 一、城乡居民和单位存款 } \\
\hline (一) 活期存款 & 0.35 & 0.35 \\
\hline \multicolumn{3}{|c|}{ (二) 整存整取定期存款 } \\
\hline 三个月 & 1.85 & 2.10 \\
\hline 半年 & 2.05 & 2.30 \\
\hline 一年 & 2.25 & 2.5 \\
\hline 二年 & 2.85 & 3.1 \\
\hline 三年 & 3.5 & 3.75 \\
\hline \multicolumn{3}{|l|}{ 二、各项贷款 } \\
\hline 一年以内(含一年) & 5.10 & 5.35 \\
\hline 一年至五年(含五年) & 5.50 & 5.75 \\
\hline 五年以上 & 5.65 & 5.90 \\
\hline
\end{tabular}




\begin{tabular}{|l|l|l|}
\hline 三、个人住房公积金贷款 \\
\hline 五年以下(含五年) & 3.25 & 3.50 \\
\hline 五年以上 & 3.75 & 4.00 \\
\hline
\end{tabular}

图 2 存款利率表

根据北京市近几年的贷款利率变化用, Matlab 做出变化折线图, 并推断 2020 年的贷款利率.

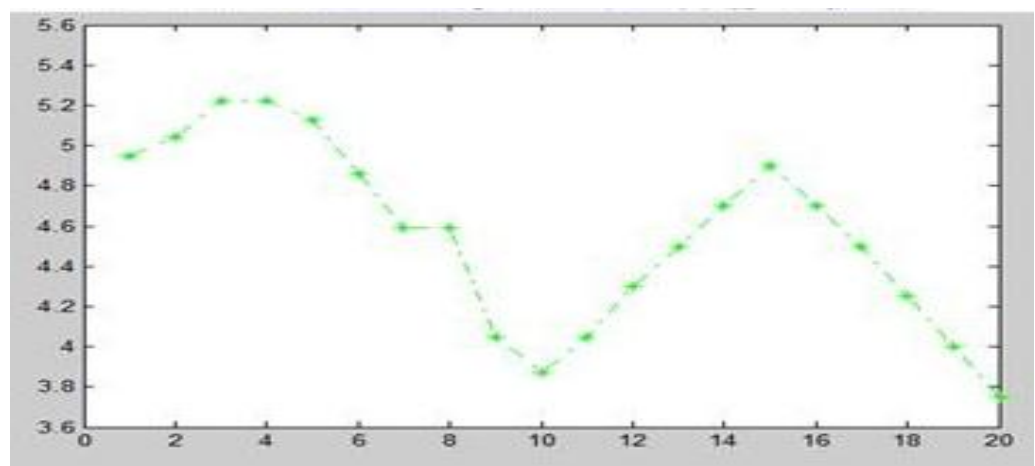

图 3 贷款利率走势图

假设 $n=c$, 所以 $Q_{a}\left[c\left(1-v_{1}\right)+d\right]<Q_{a}(n+d)$, 即 $d_{1}<d_{2}$, 实际 $n \geq c$, 正常情况, $d_{1}<c_{1}$ 。综上 所述，按照上述计算方法时在贷款买房与储蓄买房两种方式中储蓄买房更合适。

\section{2 买房出租}

此种投资方式涉及用户成本问题，用户成本由以下因素组成，即房屋作为不动产的预期利息收入、购 房所存在的借贷资金的利息、房屋使用过程中所支付的经营费用，包括折旧费、维修费、保险费、房产税 等、房地产交易成本、住宅保有期间资产价值的损益。查阅相关文献根据用户成本的有关定义, 首先, 我 们进行对用户成本的预算。则有, $d_{d}=Q_{1} c-\left[Q_{2}(1-f)-Q_{1}\right]=Q_{1}(1+c)-Q_{2}(1-f)$ 。其中 $Q_{2}(1-f)-Q_{1}$ 为房价变动所带来的资产收益。

再进一步对此分析, 可以得到, $C=D c+N n+Q_{1}(g+j+v)-\left[Q_{2}(1-f)-Q_{1}\right]$ 。其中首付资金的机 会成本、抵押贷款利息、各项必要的成本支出在实际运营中都为正项。

然后，我们对租金与用户成本的关系进行分析：

（1）当 $c \leq 0$ 即 $Q_{2}>D c+N n+\frac{Q_{1}(g+j+v)}{1-f}$ 时, 此时, 房价上涨, 前三项的支出成本远小于房产增 值的收益, 若是买房已超过五年, 可以考虑将房子卖出, 其中 $Q_{2}(1-f)-Q_{1}$ 为买房的收益。

（2）当 $c>0$ 即 $Q_{2}>D c+N n+\frac{Q_{1}(g+j+v)}{1-f}$ 时, 此时, 前三项的支出成本大于房产增值的收益, 应 该出租房屋来补充这个差价。进而，我们来分析如何来确定租金。即如何确定租金和用户成本相当。查阅 资料, 我们得到如下的计算公式: $a_{t}=\left(1+r_{1}\right)^{-1} \times\left(1+r_{2}\right)^{-1} \times \cdots \times\left(1+r_{t-1}\right)^{-1} a_{1},(\mathrm{t}=1,2,3, \ldots)$ (其中 $\mathrm{L}$ 为售


未来各期的租金之和时. 若 $L_{n}$ 为各期房价、 $V_{n}$ 为各期租金、 $c_{n}$ 为各期利率 $(\mathrm{n}=1,2,3, \cdots)$, 则有: 
$L_{1}=V_{1}+\cdots, L_{1}=V_{1}+\cdots$. 最后, 计算以上两式可得: $V=\frac{d_{d}}{1+f}$ 。这是在较为理想的状况下, 粗略的用户 成本与租金的关系, 在实际的情况下, 租金应该大于 $\frac{d_{d}}{1+f}$, 才能收回成本。

\section{3 租售比问题}

假设买房需要贷款，如北京这套房子的基本情况如下：

（1）房价：每平米 12000 元；面积：45m2；契税：16200 元; 房屋维修基金：10800 元; 装修和家用 电器: 43000 元; 合计: 61 万。

（2）首付款金额： 20 万; 约定年利率：6\%; 利息滚动方式: 年复利; 还款方式: 第 1 年一第 10 年不 付任何本息，第 11 年-第 20 年每月需要支付 2000 元，第 21 年年初一次性支付 520648 元。

（3）银行贷款金额： 41 万; 年利率：同期银行贷款利率打七折，即 $5.94 \% * 0.7=4.158 \%$; 利息滚动方 式: 月复利; 贷款年限: 30 年; 还款方式: 每月还款 1994 元。

（4）房子现租金：每年 24000 元 (水电物业等杂费由承租方另外支付）。

建立模型：计算为期 20 年的买房和租房的回报净现值。

如表 1:

表 1 各项所占比率

\begin{tabular}{|c|c|}
\hline 通货膨胀率 & $3.00 \%$ \\
\hline 房屋持有成本/房租 & $20.00 \%$ \\
\hline 首付比例 & $20.00 \%$ \\
\hline 按揭贷款利率 & $6.00 \%$ \\
\hline 自有资金投资回报率 & $3.00 \%$ \\
\hline
\end{tabular}

根据以上信息拟定二十年的付款信息，如表二:

表 2 二十年付款信息

\begin{tabular}{|c|c|c|c|}
\hline 年次 & 房租 & 银行按揭 & 差额 \\
\hline 第一年 & 24000 & 23928 & 72 \\
\hline 第二年 & 25680 & 23928 & 1752 \\
\hline 第三年 & 27477.6 & 23928 & 3549.6 \\
\hline 第四年 & 29401.03 & 23928 & 5473.03 \\
\hline 第五年 & 31459.10 & 23928 & 7531.1 \\
\hline 第六年 & 33661.24 & 23928 & 9733.24 \\
\hline$\ldots \ldots \ldots$ & $\ldots$ & ......... & $\ldots \ldots \ldots$ \\
\hline 第十一年 & 47211.57 & 23928 & -716.43 \\
\hline 第十二年 & 50516.38 & 23928 & 2588.38 \\
\hline 第十三年 & 54052.53 & 23928 & 6124.53 \\
\hline$\ldots \ldots \ldots$ & $\ldots \ldots \ldots$ & ......... & .......... \\
\hline 第二十年 & 86796.55 & 23928 & 38868.55 \\
\hline
\end{tabular}

二十年房屋合计 : 983891.81 元, 银行按揭还款合计 478560 元, 净赚 983891.81-478560-240000=26533.81 元。通过此模型，可发现买房与租房两个系数相关关量：租售比和房产升值速度。 


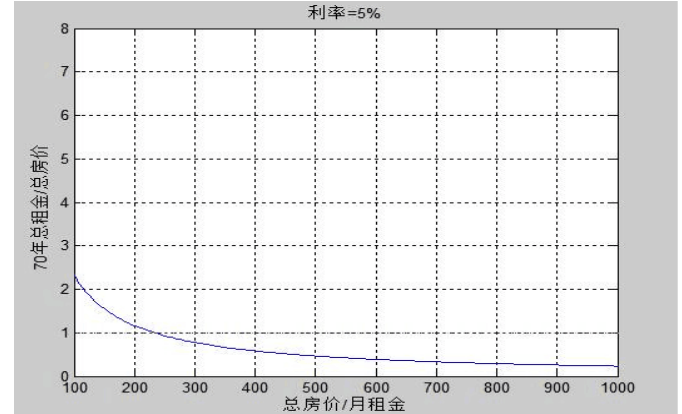

图 4 租售比

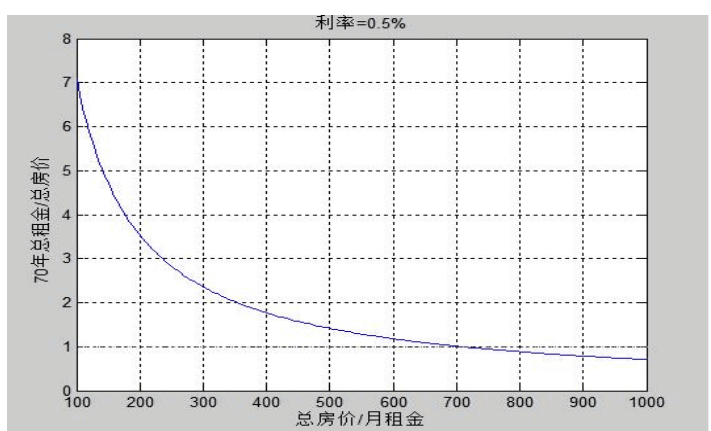

图 5 租售比

利用线性规划中的单变量求解得到了买房和租房的回报净现值相等，我们绘制了一条曲，如图 6:

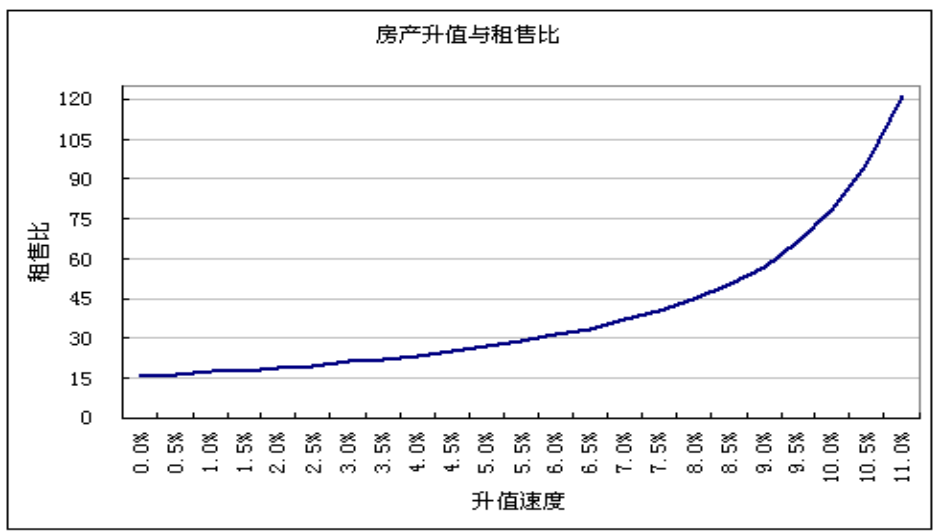

图 6 房价升值与租售比

结果分析出, 第 21 年年初, 卖掉房子收入 100 万, 一次性还清银行剩余贷款 195586, 净赚 549097.81 。 由此看出，在 2030 年一套二手房单身公寓可卖 100 万，那么 549097.81 可买一辆车。

2009 2013北京平均房价走势

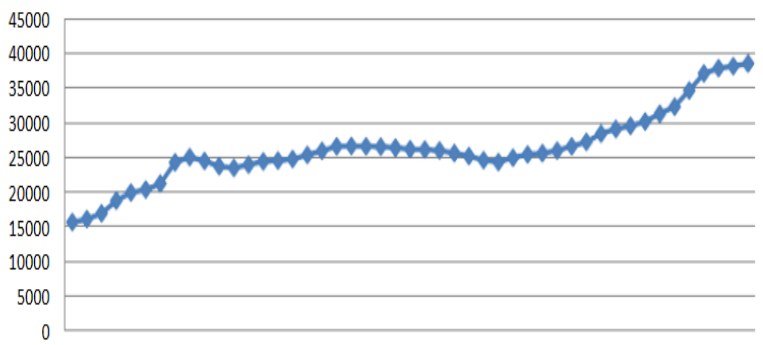

图 7 北京市平均房价走势图

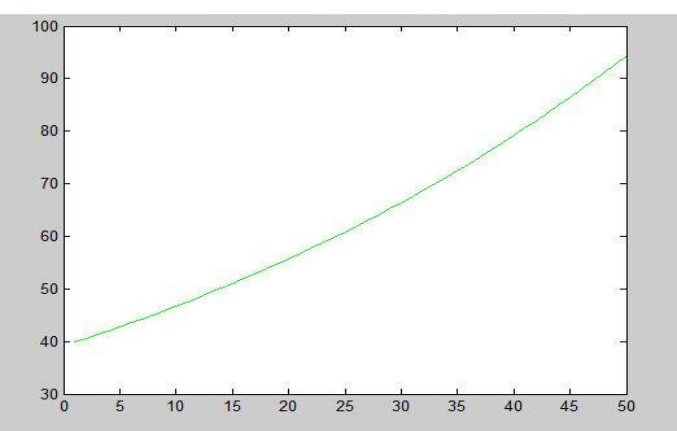

图 8 房租与房价走势图

由图 8 可看出, 未来 20 年房产达到 $9.5 \%$ 以上, 买房比租房便宜。

\section{4 小结}

通过对影响北京市买房 or 租房的各种因素的分析, 找出了最重要的几点影响因素, 建立数学模型, 根据北京市房价, 租售比, 存款利率, 贷款利率等数据, 数据真实, 贴合实际。如今买房子的认识越来越 多，本文所最后的决策方式可以为投资房产的买房者作为参考，具有很高的使用价值。

缺点: 首先, 数据在收集整理应用的过程中, 肯定会有一定的误差, 其次, 本模型在求租率和房产升 
值时处理提出了自己的看法, 抛弃了不合理的拟合, 但是比较简单, 这样不是很全面和精确。

参考文献:

[1] 刘来福, 黄海洋, 数学模型与数学建模 [M]. 北京: 北京师范大学出版社, 2009 年。

[2] 姜启源，数学模型[M]．北京：高等教育出版社，2012 年。

[3] 邓卫, 宋杨编著, 《住宅经济学》 [M], 北京, 清华大学出版社, 2008 年。

[4] 晶灿, 买房或租房你选哪种 租房价高不如贷款买房 [J]. 中外房地产导报, 2006 (10):175-178.

[5] 张江洋, 买房与租房价值比较分析 [J]. 企业经济, 2012 (1) : 151-153.

[6] 方华，厉晓敏. 买房还是租房的博交 $[J]$. 方华, 厉晓敏, 2016 (01)，65-66．

[7] 方皓彦, 雷科阳, 邱奕, 郝志强, 邱雅琪. 基于数学建模法的武汉市买房或租房分析 $[J]$, 科技创业月刊, 2016(11), 36-38 .

\section{References:}

[1] Liu Laifu, Huang Haiyang. Mathematical Model and Mathematical Modeling [M].Beijing: Beijing Normal University Press, 2009.

[2] Jiang Qiyuan. Mathematical Model [M].Beijing: Higher Education Press, 2012.

[3] Deng Wei, Song Yang authoring. <Italic $>$ Housing Economics $</$ Italic $>$ [M], Beijing: Tsinghua University Press, 2008.

[4] Jing Chan. Which Do You Prefer to, Buy a House or Rent? Renting Prices Are Higher Than Loan to Buy a House [J].Chineseand Foreign Real Estate Times, 2006(10):175-178.

[5] Zhang Jiangyang. comparative Analysis on Buying a House and Rental Value [J].Enterprise Economy,2012(1):151-153.

[6] Fang Hua, Li Xiaomin. Games Buying or renting house [J].Fang Hua, Li Xiaomin, 2016(01), 65-66.

[7] Fang Haoyan, Lei Keyang, Qiu Yi, Hao Zhiqiang, Qiu yaqi. analysis on buying or renting house in Wuhan City based on mathematical modeling [J],Pioneering With Science \& Technology Monthly,2016(11),36-38. 